\title{
Relationship between Pain Behavior and Changes in KCNA2 Expression in the Dorsal Root Ganglia of Rats with Osteoarthritis
}

\author{
Qihong Zhao $\mathbb{D},{ }^{1,2}$ Lei Fan, ${ }^{3}$ Jiafeng Wang $\mathbb{D}^{2},{ }^{2}$ and Xiaoming Deng $\mathbb{D}^{2}$ \\ ${ }^{1}$ Department of Anesthesiology, The Second Affiliated Hospital, Bengbu Medical College, Bengbu, Anhui, China \\ ${ }^{2}$ Department of Anesthesiology and Intensive Care, The First Affiliated Hospital, \\ The Naval Military Medical University (The Second Military Medical University), Shanghai, China \\ ${ }^{3}$ Department of Orthopedics, The Third Affiliated Hospital, Sun Yat-sen University, Guangzhou, Guangdong, China \\ Correspondence should be addressed to Qihong Zhao; zhaoqh0516@163.com and Xiaoming Deng; deng_x@yahoo.com
}

Received 3 August 2019; Revised 28 April 2020; Accepted 19 June 2020; Published 18 July 2020

Guest Editor: Jun Zou

Copyright () 2020 Qihong Zhao et al. This is an open access article distributed under the Creative Commons Attribution License, which permits unrestricted use, distribution, and reproduction in any medium, provided the original work is properly cited.

\begin{abstract}
Objective. To investigate the relationship between pain behavior and potassium voltage-gated channel subfamily A member 2 (KCNA2) expression in dorsal root ganglia (DRGs) of rats with osteoarthritis (OA). Methods. Male Sprague-Dawley rats were randomly divided into three groups: blank control group (group C), normal saline group (group S), and group OA. Paw withdrawal mechanical threshold (PWMT) and paw withdrawal thermal latency (PWTL) were measured one day before injection and one, two, four, and six weeks after injection. At one, two, four, and six weeks after injection, pathological knee joint changes and activated transcription factor-3 (ATF-3) and KCNA2 expressions in DRGs were analyzed. Results. Compared with preinjection, PWMT and PWTL at two, four, and six weeks after injection were significantly decreased in the group OA $(P<0.05$ or 0.01). Compared with group C, PWMT and PWTL at two, four, and six weeks after injection were significantly decreased in the group OA $(P<0.05$ or 0.01$)$. In the group OA, slight local articular cartilage surface destruction was found at week one. The cartilage surface destruction gradually developed, and the exacerbation of cartilage matrix reduction and bone hyperplasia were increasingly aggravated and eventually evolved into advanced OA in the second to sixth weeks. Compared with group C, ATF-3 expression was significantly increased, and KCNA2 expression was significantly decreased in the group OA at two, four, and six weeks after injection $(P<0.05$ or 0.01$)$. Compared to baseline, ATF-3 expression was significantly increased, and KCNA2 expression was significantly decreased in the group OA $(P<0.05$ or 0.01$)$. Conclusion. Pain behavior in OA rats was associated with decreased KCNA2 expression in DRGs.
\end{abstract}

\section{Introduction}

$\mathrm{OA}$ is a common disease in the aging population which affects bones and motor system; its incidence rate in people who are 65 years old and above is more than $50 \%$, and its prevalence rate in people who are 75 years old and above is approximately $80 \%$ [1]. Pain is the most important symptom of $\mathrm{OA}$, and it is also the main cause that affects the human health and causes disability $[1,2]$. However, little is known about the pathogenesis of OA pain, and thus, there is a lack of targeted treatment and measures to control OA pain. Nonsteroidal anti-inflammatory drugs $[3,4]$ and opioids $[5,6]$ have a certain effect on relieving OA pain, but longterm use of these drugs has great side effects, and they cannot fundamentally reverse or delay the progression of the disease [7-10]. Although surgery is effective in relieving pain in patients with advanced OA, surgery itself can cause pain and increase the financial burden on the patient. Therefore, studying the pathogenesis of OA pain and exploring the possible targets for prevention and treatment of $\mathrm{OA}$ pain have a great research value and clinical significance.

Studies have shown that OA causes not only changes in the local nerve distribution $[11,12]$ but also damage to the DRGs [11, 13]. However, increased excitability of neurons in DRGs caused by injury is an important mechanism of chronic pain. The results of some researches [14-16] have confirmed that OA pain has similar characteristics as neuropathic pain (NP), but whether the mechanism of OA 
pain is similar to that of NP remains unclear. Recent studies have indicated that downregulation and dysfunction of KCNA2 are one of the important pathogeneses of NP $[17,18]$. Furthermore, other researches have demonstrated that changes in the expression and function of voltage-gated sodium channel in DRGs are related to hyperalgesia in OA pain $[19,20]$. Therefore, we hypothesized that KCNA2 in DRGs is related to changes in pain behavior in OA animal models. This paper aims to study the relationship between pain behavior and KCNA2 expression in DRGs of rats with $\mathrm{OA}$ and to provide a theoretical basis for further exploring the pathogenesis of OA pain.

\section{Materials and Methods}

2.1. Animals and Grouping. As the decrease of estrogen is an important pathogenesis of osteoarthritis, we choose male rats in order to eliminate the interference of estrogen to animal model. A total of 144 male Sprague-Dawley rats (aged 7-8 weeks, weight 230-270 g) were acquired from Guangdong Experimental Animal Center (qualified number: 44007200052028). Then, they were randomly equally divided into the following three groups: blank control group (group C), normal saline group (group S), and group OA.

2.2. Model Establishment. According to literatures [21-24] and slightly modified techniques, intraperitoneal injection of $6 \%$ sodium pentobarbital was administered in rats $(60 \mathrm{mg} / \mathrm{kg})$. After sufficient anesthesia, fur on the left knee joint was shaved off, disinfected by medical iodine, and then deiodinated by $75 \%$ ethanol. The puncture needle was inserted into the joint cavity through the lateral part of the infrapatellar ligament. The group OA received intra-articular injection of $4 \%$ monosodium iodoacetate (MIA) (Macklin Biochemical Technology Co. Shanghai, China) $2 \mathrm{mg}$ (dissolved in $50 \mu \mathrm{L}$ normal saline) [21-23], group $S$ was injected with the same volume of normal saline, and group $\mathrm{C}$ was only subjected to articular cavity puncture without injection.

\subsection{Observation Indicators}

2.3.1. Pain Behavior. After preadapting for $30 \mathrm{~min}$, pain behavior in rats was measured one day before intra-articular injection and one, two, four, and six weeks after injection. Based on references $[17,25,26]$ and slightly altered, Aesthesio Von-Frey filaments (Ugo Basile, Gemonio, Italy) with different folding forces $(2,4,6,8,10,15,26$, and $60 \mathrm{~g})$ were applied to stimulate the midpoint of the left paw. The filament was applied for a period of $\leq 4 \mathrm{~s}$, and the procedure was repeated five times at $30 \mathrm{~s}$ intervals, and three or more positive reaction fold forces were recorded as withdrawal mechanical threshold (PWMT). According to some other studies $[17,27,28]$, the left paw of the rat was irradiated with a laser heat source lamp of infrared thermal pain tester (RWD, San Diego, USA) at a temperature of $55^{\circ} \mathrm{C}$. The time from the start of irradiation to the foot-licking phenomenon in the rat was recorded. A cut-off time of $60 \mathrm{~s}$ was used to avoid tissue injury. The irradiation was repeated three times at $10 \mathrm{~min}$ intervals, and the average value of three measurements was considered as paw withdrawal thermal latency (PWTL).

2.3.2. Knee Joint Histopathology. The left knee joint tissue was obtained after injection into the articular cavity at one, two, four, and six weeks, fixed in $4 \%$ paraformaldehyde for $48 \mathrm{~h}$, and then placed in a decalcified solution. The fluid was changed every three days, and the articular tissue was cut into $8-\mu \mathrm{m}$-thick paraffin sections for use after two weeks. The above paraffin sections were dewaxed and hydrated for further dyeing. Hematoxylin-Eosin staining (Biyuntian Biotechnology Research Institute, China) was performed, and it included the following steps: adding Hematoxylin dyeing solution for $10 \mathrm{~min}$, 1\% hydrochloric acid alcohol differentiation after water wash for 5-10 s, and adding eosin dyeing solution for 1-3 min. Safranin O-Fast Green staining (Beijing Baiao Laibo Technology Co., Ltd., China) was performed, and it included the following steps: Hematoxylin staining for $5 \mathrm{~min}$, hydrochloric acid alcohol differentiation after water wash for 5-10 s, washing and then add strengthening green solution for $3 \mathrm{~min}$, quick rinsing with $1 \%$ acetic acid for $10-15 \mathrm{~s}$, and staining with Safranin O solution for 2-3 min. The stained sections were dehydrated, waxed, and sealed, and then they were observed under a microscope.

2.3.3. Expression of ATF-3 in DRGs. The animals were killed at one, two, four, and six weeks after the intra-articular injection: the rat's thoracic cavity was cut open quickly, the left ventricle was inserted, and the right atrium was cut open, and $4 \%$ paraformaldehyde was injected for fixation. The DRGs at L3-5 of the left lumbar vertebra of the rat were obtained for frozen section. The sections were rewarmed at room temperature for $30 \mathrm{~min}$. Then, the sections were blocked by the rupture solution containing $0.2 \%$ Triton $\mathrm{X}-100$ and $5 \%$ goat serum at room temperature for $2 \mathrm{~h}$, and the corresponding primary antibodies of rabbit-anti-rat ATF-3 (GTX129120, 1:200, GeneTex, Radnor, USA) and mouse anti-rat NeuN (GTX30773, $1: 200$, GeneTex, Radnor, USA) were added dropwise at $4^{\circ} \mathrm{C}$ overnight. On the next day, the sections were removed and rinsed, and the corresponding fluorescent secondary antibodies of donkey antirabbit (AB141637, $1: 1000$, Thermo Fisher, Waltham, USA) and goat-anti-mouse (ab150113, 1:1000, Abcam, London, $\mathrm{UK}$ ) were added; they were incubated for $2 \mathrm{~h}$ at room temperature and stained with Hoechst 33342 (Sigma, St. Louis, USA) for $10 \mathrm{~min}$. On observation by confocal microscopy after the sections were sealed, the neurons were green, ATF-3 were red, and image analysis was performed using Image J software (NIH, MA, USA).

2.3.4. Expression of KCNA2 in DRGs. The L3-5 DRGs tissue on the left side of the rat was obtained at one, two, four, and six weeks after articular cavity injection and stored in a refrigerator at $-80^{\circ} \mathrm{C}$. All samples were cut to pieces, added with tissue lysis fluid, fully homogenized on ice, and lysed. 
Then, they were centrifuged at $12,000 \mathrm{r} / \mathrm{min}$ for $30 \mathrm{~min}$ at $4^{\circ} \mathrm{C}$. The supernatant was obtained, and the protein concentration was determined by the bicinchoninic acid assay balanced. Then, it was denatured in boiling water and stored in a refrigerator at $-20^{\circ} \mathrm{C}$ for use. The same amount of protein samples was obtained for electrophoresis. The proteins were separated and transferred to a polyvinylidene fluoride membrane, and it was blocked by $5 \%$ skim milk at room temperature for $1 \mathrm{~h}$. Rabbit-anti-rat KCNA2 polyclonal antibody (GTX54835, 1:1000, GeneTex, Radnor, USA) was added dropwise, incubated at $4^{\circ} \mathrm{C}$ overnight, and rinsed after removal. Then, horseradish peroxidaseconjugated goat-anti-rabbit secondary antibody (ZDR-5118, $1: 1000$, Zhongshan Jinqiao Biotechnology Co., Ltd., Beijing, China) was added and incubated for $2 \mathrm{~h}$ at room temperature. ECL Western Blotting Substrate kit (Thermo Fisher, Waltham, USA) was used for color rendering, and analysis was performed by Image J Software.

2.3.5. Statistical Analysis. All data were analyzed with SPSS 20.0 statistical software package (SPSS Inc., IL, USA) and presented as mean \pm standard deviation (SD). All data were graphed by Excel 2010 (Microsoft, MA, USA). The pain behavior data were analyzed by the repeated-measures analysis of variance (ANOVA). The comparison between groups of PWMT and PWTL was performed respectively by the multiplex analysis and the simple effect test, and intragroup comparison was performed using the least-significant difference (LSD) test. The remaining data were analyzed by the one-way ANOVA followed by the LSD test. $P<0.05$ was considered statistically significant.

\section{Results}

3.1. Changes in Pain Behavior in the Three Groups of Rats. Compared with baseline, PWMT and PWTL were significantly lower in the group OA $(P<0.05$ or 0.01$)$ at two, four, and six weeks after intra-articular injection; PWMT and PWTL were lowest at four weeks $(P<0.01)$. There was no significant difference in PWMT and PWTL between four and six weeks after OA model establishment $(P>0.05)$. Compared with group C, at two, four, and six weeks, PWMT and PWTL were significantly decreased in the group OA $(P<0.05$ or 0.01$)$ (see Figure 1$)$.

\subsection{Histopathological Changes in the Knee Joints in the Three} Groups of Rats. In group OA, the local cartilage surface was incomplete and opaque, the proteoglycan in the cartilage matrix was uniformly stained red, the subchondral cortical bone and trabecular bone were uniformly stained green, and the tidal line was clear at one week. At two weeks, cartilage surface damage was aggravated. It was observed that the cartilage matrix was reduced, the subchondral cortical bone and trabecular bone were increased, and some tidal lines were blurred. At four weeks, cartilage damage was further aggravated, local bone was exposed, the joint cavity was narrow, the cartilage matrix was reduced greatly, the extent of subchondral cortical bone and trabecular bone was further expanded than that at two weeks, and the tidal line was unclear. At six weeks, the articular cartilage was severely damaged, the exposed bone surface was augmented, the cartilage matrix was extensively decreased, the subchondral cortical bone and trabecular bone were enlarged, and part of the bone was convex to the joint cavity. In group $C$, the articular cartilage surface was intact and smooth at one, two, four, and six weeks, with clear articular chondrocytes and a visible tidal line and absence of hyperosteogeny (Figure 2).

\subsection{Expression of ATF-3 in DRGs in the Three Groups of Rats.} In order to determine whether the DRGs of rats with OA were involved, L3-5 DRGs on the same side as intra-articular injection were collected for analysis of the ATF-3 expression. The results showed that the expression of ATF-3 in DRGs was significantly increased at two, four, and six weeks after the injection with MIA compared to before the injection $(P<0.05$ or 0.01$)$, and compared with group $C$, the expression of ATF-3 in DRGs in the group OA was significantly increased at two, four, and six weeks after the intraarticular injection $(P<0.05$ or 0.01$)$ (Figure 3$)$.

\subsection{Expression of KCNA2 in DRGs in the Three Groups of Rats.} We further explored the expression of KCNA2 in DRGs. The results indicate that the expression of KCNA2 was significantly decreased at two, four, and six weeks after intra-articular administrated with MIA than baseline $(P<0.05$ or 0.01 ), and compared with group $C$, the expression of KCNA2 in DRGs in the group OA was significantly decreased at two, four, and six weeks $(P<0.05$ or 0.01$)$ (Figure 4$)$.

\section{Discussion}

$\mathrm{OA}$ is a common and frequently observed disease in the clinic, and pain is the main reason that affects the life of patients. However, the mechanism of OA pain is very complicated, and little is known about this mechanism at present, which results in certain difficulties in the prevention and treatment of OA.

4.1. Scientific and Reasonable Establishment of Animal Model. Intra-articular injection of MIA into the unilateral knee joint in rats destroyed the articular cartilage, the synovial membrane, and ligament to some extent and changed the original stability of the joint, thus leading to the development of OA. These pathological changes are similar to those in human OA. Because chronic deterioration of joints is associated with chronic pain behavior, this model can assess the changes in pain in animal models. The model has a short modeling period, is stable and reliable, requires a simple operation, and has a high success rate. Therefore, it is widely used in OA research $[24,29,30]$. According to several previous studies $[11,21-23,31,32]$, we selected the dose, concentration, and volume of MIA. This study showed that pathological changes occurred in the knee joint of rats in the 


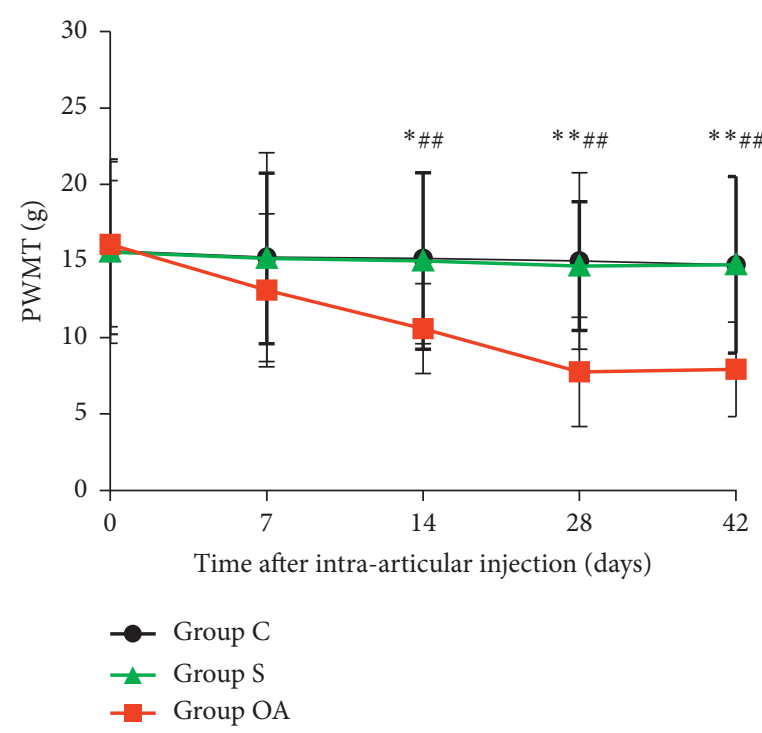

(a)

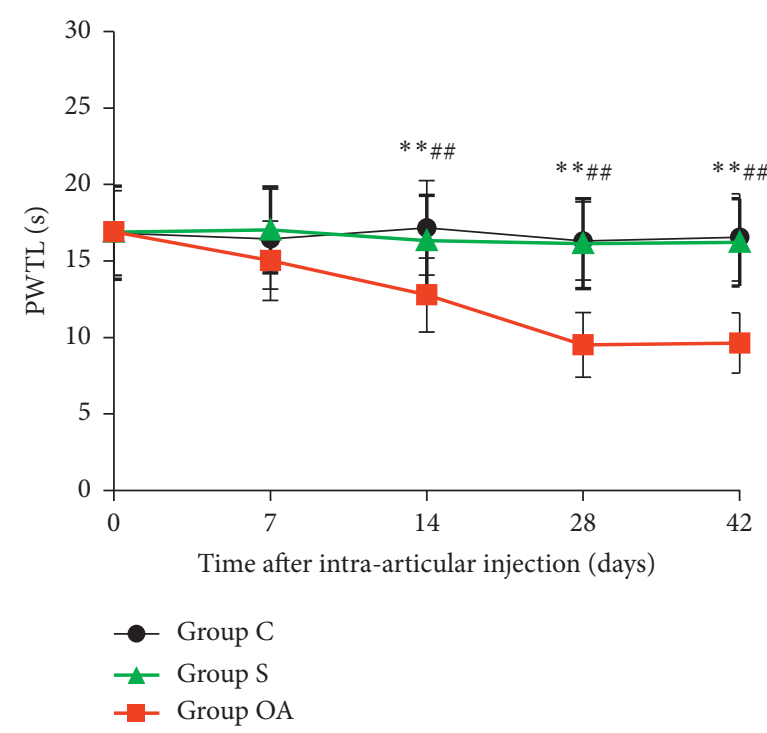

(b)

FIGURE 1: Comparison of PWMT (a) and PWTL (b) in the three groups of rats at each time point. Each symbol represents mean \pm SD (standard deviation), $n=12$ per group; ${ }^{*} P<0.05,{ }^{* *} P<0.01$ vs. group $C$; ${ }^{\#} P<0.05$, ${ }^{\# \#} P<0.01$ vs. baseline in the OA group. Repeatedmeasures ANOVA: (a) multiple groups; (b) multiple groups $\times$ time.

OA group within one week after intra-articular injection of MIA, and the pathological changes after four weeks were similar to those in advanced human OA. Furthermore, pain behavioral changes of rats were apparent in group OA, indicating that the OA model was successfully established.

\subsection{Changes of Pain Behavior in OA Model Rats.} Fernihough et al. [24] reported that, compared with partial medial meniscectomy, the pain behavioral changes in the knee joint OA model constructed by intra-articular MIA injection were more similar to those in OA patients. Furthermore, another study provided evidence that both MIA and meniscal transection models in rats are similar to those of human knee OA, but inflammation may play a greater role in the development of pain behavior in the meniscal transection model [33]. Kim et al. [29] reported that, compared with the saline group, the PWMT and PWTL in the OA group were significantly decreased. The above results suggest that the characteristic of OA pain is similar to that of NP. Pain hypersensitivity and pain allergies are the two prominent symptoms of NP. This study showed that PWMT and PWTL of rats in the group OA were significantly decreased than those in group $\mathrm{C}$ after two weeks of intraarticular injection of MIA, and they reached a peak at four weeks after intra-articular injection, suggesting that OA pain had similar characteristics to NP and that pain allergy and hypersensitivity were involved in the pathogenesis of OA pain.

4.3. The Involvement of DRGs in Changes of Pain Behavior in OA Rats. The DRGs serve as a relay station for the pain signal transmission process. When a peripheral nerve is injured, the DRGs, as the first level neurons, receive the incoming pain signal and transmit it to the dorsal horn of the spinal cord and higher centers. Furthermore, Albayrak et al. reported that pulsed radiofrequency therapy to DRG contributes to reducing the degree of pain in patients with persistent postsurgical pain after total knee arthroplasty [34]. Therefore, DRGs play an important role in the pathogenesis of OA pain $[13,35]$. Joint inflammation is a major clinical problem and a main cause of debilitating chronic pain, characterized by pronounced mechanical hyperalgesia and persistent pain at rest [36]. Pain mechanisms require the sensitization of primary sensory neurons, whose cell bodies are located in DRG, and involve several media-tors that trigger particular signal-transduction pathways. A great part of these pathways imply the activation of transcription factors, in which gene expression becomes altered. In particular pathological conditions, DRGs are actually responsible for the synthesis of signaling molecules involved in reaction cascades that can ultimately lead to phenomena like survival or regeneration [37]. Activating transcription factor 3 (ATF-3) has been suggested as acting as an "adaptive response" due to its ability to respond differently according to the cellular context [38]. ATF-3, which shows low expression under normal conditions, increases rapidly when the cells are stimulated by various pathophysiological processes [39]. It is the earliest transcription factor that is expressed during a cellular stress response, and its increased expression often indicates tissue damage. Its inclusion in anti- and proapoptosis mechanisms [40], cell survival [41], regeneration [42], and neuroprotection [43] signaling events has been reported. In a p-Akt-mediated survival pathway, the ATF-3/c-Jun heterodimer has been suggested to promote nerve elongation and inhibit apoptosis in neurons under death stress such as nerve injury [44]. Nowadays, ATF-3 is mostly assumed as a neuronal injury marker, after 


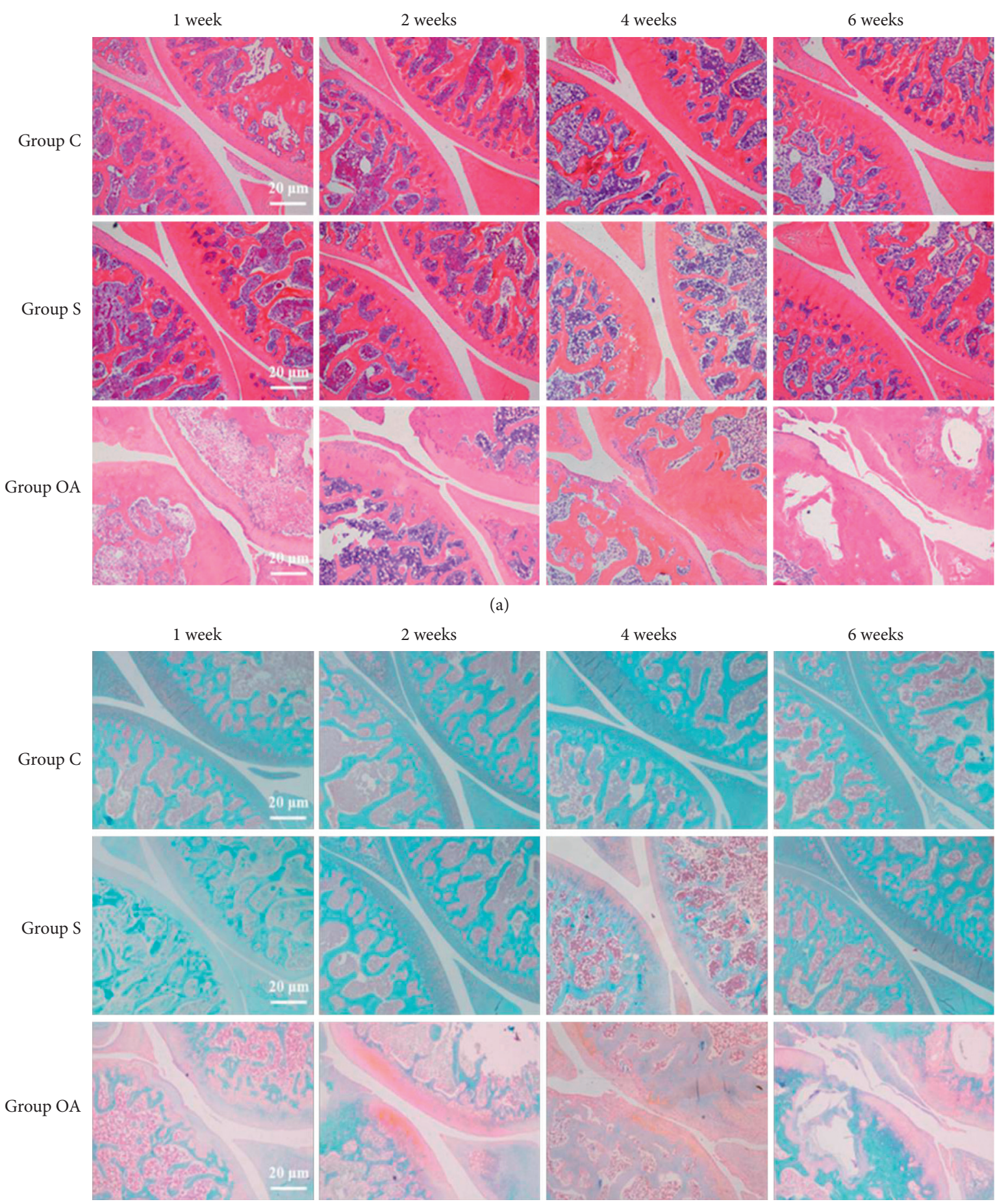

(b)

FIgURE 2: Pathological changes in the knee joints. (a) Hematoxylin-Eosin (HE) staining. (b) Safranin O-Fast Green staining. In group C, the articular cartilage surface was smooth and there was no crack formation, the cells were normal and arranged regularly, the tidal line was clear, and there was no bone hyperplasia. In the group OA, with the development of the OA, the destruction of cartilage surface is aggravated and even bone exposure occurred, the increasing exacerbation of cartilage matrix reduction and bone hyperplasia was apparent, the tidal line gradually blurred, and pathological changes showed typical advanced OA manifestations at four to six weeks.

its expression was found to be highly induced in several models of neuropathic pain [45-47]. The results of our study showed that ATF-3 expression was significantly increased in
DRGs of rats after two weeks of intra-articular injection with MIA into the knee joint, thus indicating that the neurons were damaged. However, data on ATF-3 expression in 


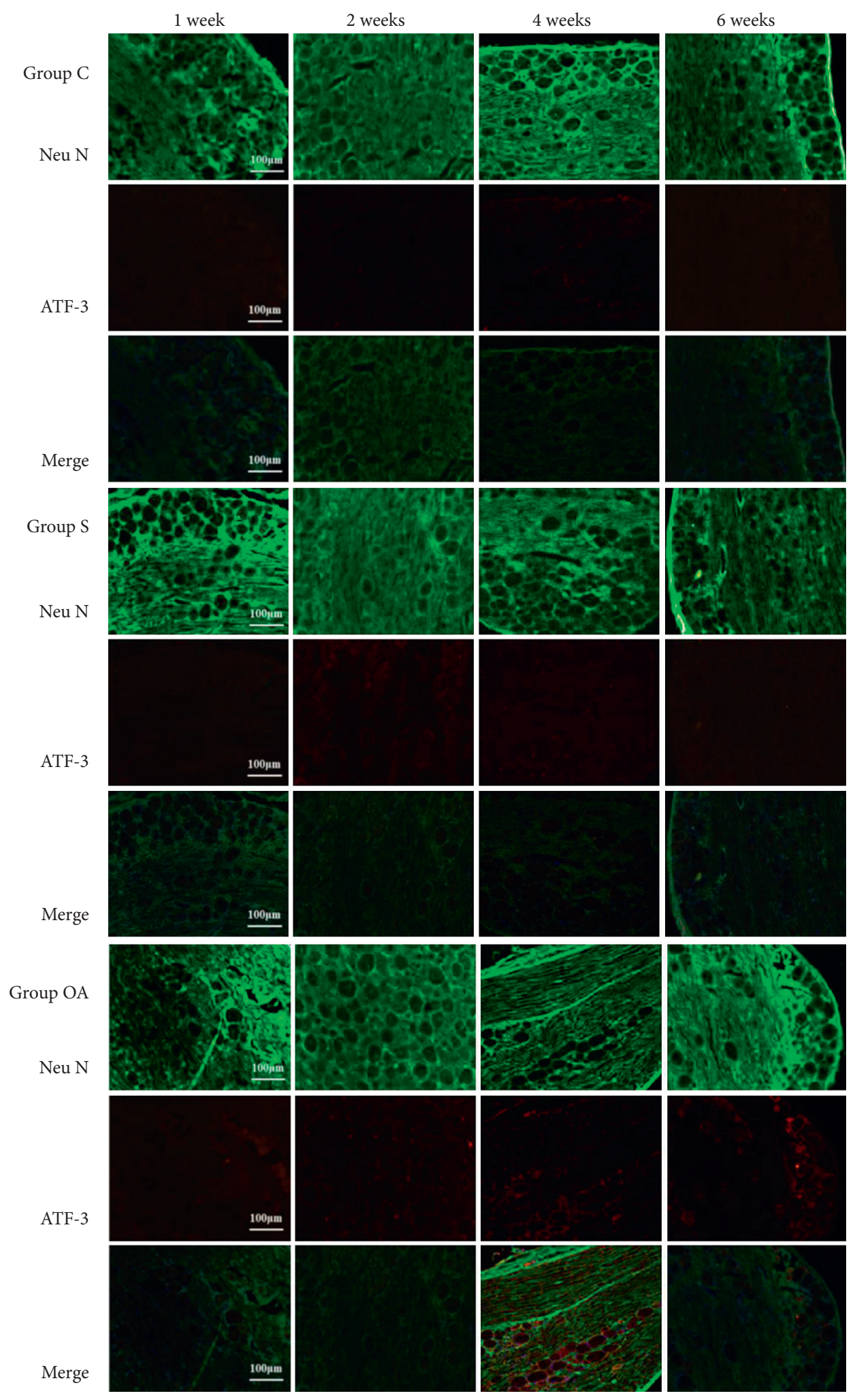

(a)

Figure 3: Continued. 

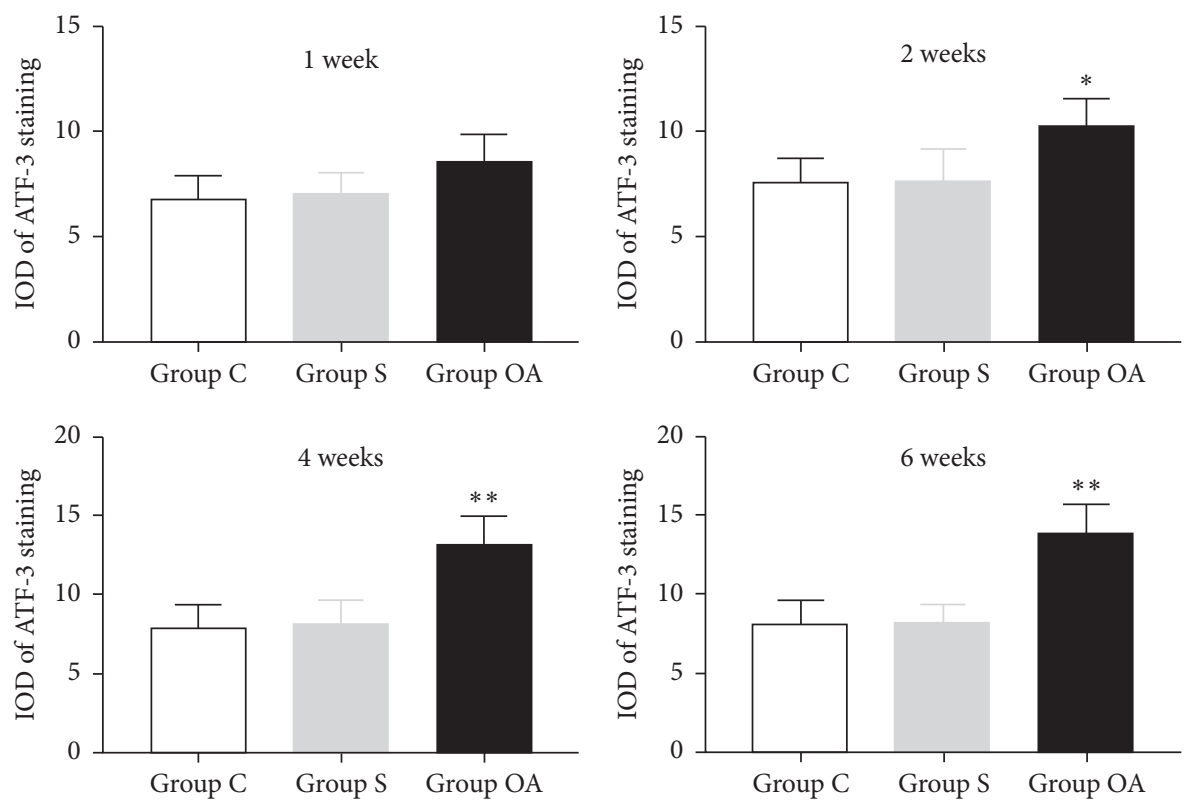

(b)

FIgURE 3: Comparison of ATF-3 expression in the three groups of rats at each time point. (a) Double immunofluorescence staining of ATF-3 in the DRGs. (b) Analysis of integrated optical density (IOD) of ATF-3. Each symbol represents mean \pm SD (standard deviation), $n=4$ per group; ${ }^{*} P<0.05,{ }^{* *} P<0.01$ vs. group C. One-way ANOVA and LSD test.

A

KCNA2

GAPDH

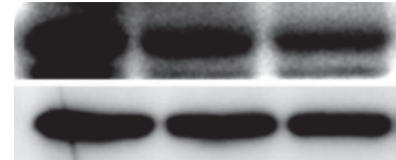

$57 \mathrm{kDa}$

$36 \mathrm{kDa}$

B

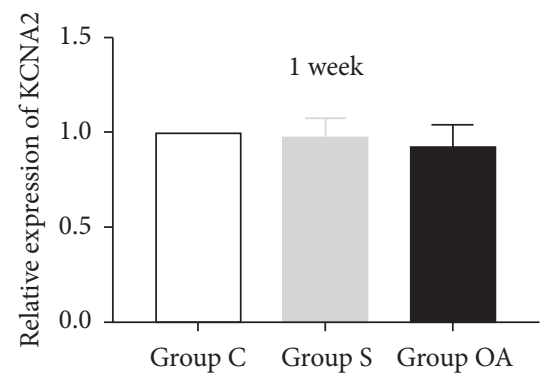

A

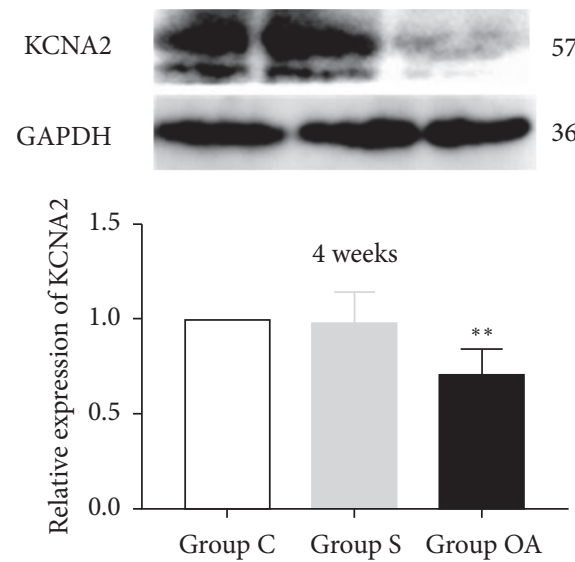

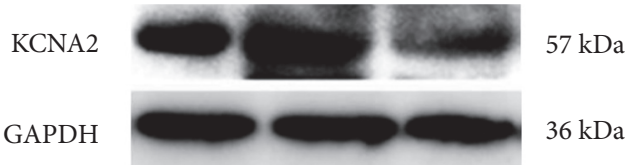
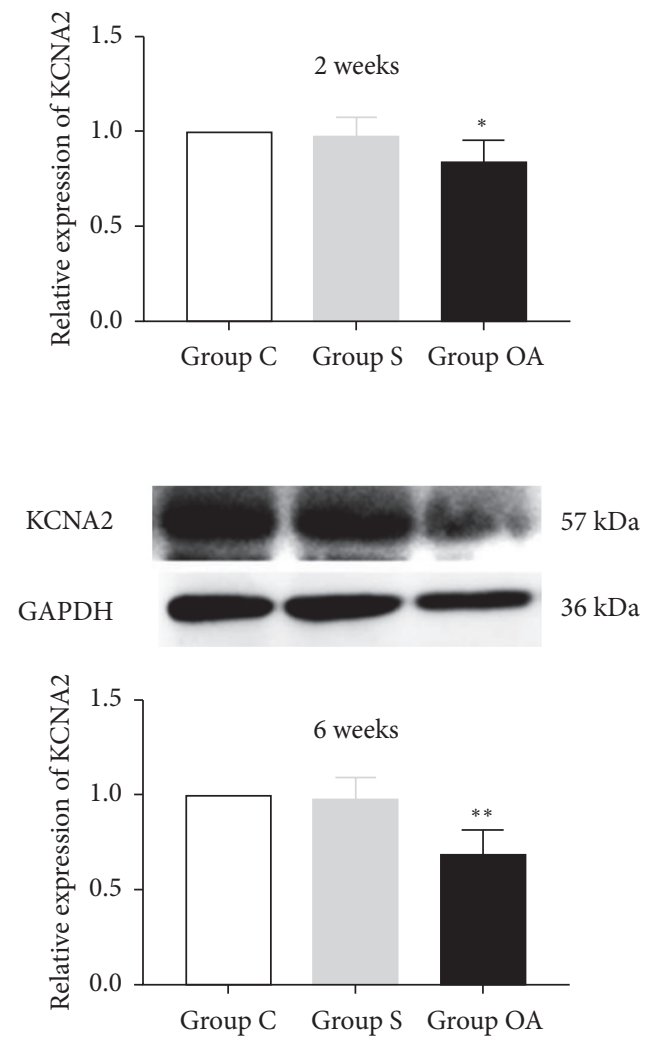

Figure 4: Comparison of KCNA2 expression in the three groups of rats at each time point. (a) Expression of KCNA2 in the DRG imaged by Western blotting. (b) Analysis of expression of KCNA2. Each symbol represents mean \pm SD (standard deviation), $n=4$ per group; ${ }^{*} P<0.05$, ${ }^{* *} P<0.01$ vs. group C. One-way ANOVA and LSD test. 
primary afferents in different models of inflammatory pain have not always been consistent [48-50]. For example, intraplantar injection of complete Freund's adjuvant (CFA) did not induce any ATF-3 expression, suggesting there is no neuronal damage associated [48, 49]. Interestingly, in the collagen-induced arthritis and monoiodoacetate-induced osteoarthritis joint pain models, ATF-3 was also expressed in DRG neurons [51, 52], suggesting some degree of neuronal damage is occurring. Taking into account these controversial data and the sparse information available on the role of ATF3 in pain processing, its expression was shown to be either increased or decreased depending on the neuronal populations, indicating ATF-3-expressing cells' size distribution and colocalization with isolectin B4 (IB4) or calcitonin generelated peptide (CGRP) [50].

4.4. The Relationship between the Expression of KCNA2 in DRG Neurons and Pain Behavior in OA Model Animals. Injury to the neurons in DRGs may cause changes in the expression or function of ion channels on the membrane $[17,20]$. The Kv channel is one of the important ion channels that play important roles in maintaining the resting membrane potential and action potential repolarization, and it is closely related to the excitability of neurons. The $\mathrm{Kv}$ channel is a tetramer composed of the $\alpha$ subunit superfamily and the $\beta$ auxiliary subunit. Kv channels can be divided into 12 subfamilies according to the $\alpha$ subunit, $1-12$, where, in the $\alpha$ subunit, Kv1.2 (KCNA2) of the Kv1 subfamily is highly expressed in DRGs [53, 54]. Decreased expression of KCNA2 in DRGs contributes to hyperalgesia in NP and increases the expression of KCNA2 to attenuate the symptoms of NP [17]. Recently, Zhao et al. [18] demonstrated that, in the chronic pain rat model created by spinal nerve ligation, the expression of KCNA2 in DRGs was significantly decreased, and the Kv current was decreased, which caused abnormal excitation and discharge of neurons and led to peripheral sensitization of pain. The results of our study showed that the expression of KCNA2 in DRGs of rats in the group OA was decreased compared with that of rats in group $\mathrm{C}$ since two weeks after MIA injection, and the difference was most significant at four to six weeks. Since the changes in pain behavior were basically consistent with the trend of $\mathrm{KCNA} 2$, we believe that the pain behavior of OA rats was related to the decrease in KCNA2 expression in DRGs. However, this study did not further study the changes of pain behavior in OA model rats after reversing the downregulation of KCNA2 expression in DRGs. In addition, there are many potential mechanisms that cause the change of KCNA2 expression in DRG neurons in other chronic pain animal models $[18,55,56]$, and this is not clear in OA model animals. However, we have not carried out further research in this field. In the future, we will conduct more in-depth research on the previously mentioned issues.

\section{Summary}

In conclusion, this study confirmed that the nature of OA pain is similar to that of NP and demonstrated the occurrence and development of OA pain are associated with the decreased expression of KCNA2 in DRG neurons.

\section{Data Availability}

The data used to support the findings of this study are available from the corresponding author upon request.

\section{Conflicts of Interest}

The authors declare that they have no conflicts of interest.

\section{Acknowledgments}

The authors would like to acknowledge the financial support from the Department of Science and Technology of Guangdong Province. This study was supported by the Foundation of Guangdong Science and Technology Assistance for Xinjiang Project (no. 2018YJ023).

\section{References}

[1] N. Arden and M. C. Nevitt, "Osteoarthritis: epidemiology," Best Practice \& Research Clinical Rheumatology, vol. 20, no. 1, pp. 3-25, 2006.

[2] T. Vos, A. D. Flaxman, M. Naghavi et al., "Years lived with disability (YLDs) for 1160 sequelae of 289 diseases and injuries 1990-2010: a systematic analysis for the global burden of disease study 2010," The Lancet, vol. 380, no. 9859, pp. 2163-2196, 2012.

[3] G. Honvo, V. Leclercq, A. Geerinck et al., "Safety of topical non-steroidal anti-inflammatory drugs in osteoarthritis: outcomes of a systematic review and meta-analysis," Drugs and Aging, vol. 36, no. Suppl 1, pp. 45-64, 2019.

[4] M. S. M. Persson, J. Stocks, D. A. Walsh, M. Doherty, and W. Zhang, "The relative efficacy of topical non-steroidal antiinflammatory drugs and capsaicin in osteoarthritis: a network meta-analysis of randomised controlled trials," Osteoarthritis Cartilage, vol. 26, no. 12, pp. 1575-1582, 2018.

[5] J. E. Pope, "Prescribing opioids for severe hip and knee osteoarthritis varies widely in the United States: the devil is in the details," Arthritis Rheumatol, vol. 71, no. 5, pp. 659-661, 2019.

[6] E. E. Krebs, A. Gravely, S. Nugent et al., "Effect of opioid vs nonopioid medications on pain-related function in patients with chronic back pain or hip or knee osteoarthritis pain: the SPACE randomized clinical trial," The Journal of the American Medical Association, vol. 319, no. 9, pp. 872-882, 2018.

[7] S. Durg, M. Lobo, L. Venkatachalam, G. Rao, and J. Bhate, "A systematic review and meta-analysis of oxaceprol in the management of osteoarthritis: an evidence from randomized parallel-group controlled trials," Pharmacological Reports, vol. 71, no. 2, pp. 374-383, 2019.

[8] K. Yoshida and R. Desai, "Unraveling the role of NSAIDs in the link between osteoarthritis and cardiovascular disease via causal mediation analysis-a guide to interpretation," Arthritis \& Rheumatology, vol. 71, no. 11, pp. 1776-1779, 2019.

[9] C. J. Hayes, N. Payakachat, and C. Li, "Evaluation of opioid use among patients with back disorders and arthritis," Quality of Life Research, vol. 27, no. 11, pp. 3021-3035, 2018.

[10] J. B. Thorlund, A. Turkiewicz, D. Prieto-Alhambra, and M. Englund, "Opioid use in knee or hip osteoarthritis: a 
region-wide population-based cohort study," Osteoarthritis Cartilage, vol. 27, no. 6, pp. 871-877, 2019.

[11] M. Thakur, W. Rahman, C. Hobbs, A. H. Dickenson, and D. L. H. Bennett, "Characterisation of a peripheral neuropathic component of the rat monoiodoacetate model of osteoarthritis," PLoS One, vol. 7, no. 3, Article ID e33730, 2012.

[12] M. Ikeuchi, Q. Wang, M. Izumi, and T. Tani, "Nociceptive sensory innervation of the posterior cruciate ligament in osteoarthritic knees," Archives of Orthopaedic and Trauma Surgery, vol. 132, no. 6, pp. 891-895, 2012.

[13] S. Miyamoto, J. Nakamura, S. Ohtori et al., "Pain-related behavior and the characteristics of dorsal-root ganglia in a rat model of hip osteoarthritis induced by mono-iodoacetate," Journal of Orthopaedic Research, vol. 35, no. 7, pp. 1424-1430, 2017.

[14] D. Bevilaqua-Grossi, M. Zanin, C. Benedetti, L. Florencio, and A. Oliveira, "Thermal and mechanical pain sensitization in patients with osteoarthritis of the knee," Physiotherapy Theory and Practice, vol. 35, no. 2, pp. 139-147, 2019.

[15] P. Moss, H. A. E. Benson, R. Will, and A. Wright, "Patients with knee osteoarthritis who score highly on the pain detect questionnaire present with multimodality hyperalgesia, increased pain, and impaired physical function," The Clinical Journal of Pain, vol. 34, no. 1, pp. 15-21, 2018.

[16] J. D. Power, A. V. Perruccio, R. Gandhi et al., "Neuropathic pain in endstage hip and knee osteoarthritis: differential associations with patient-reported pain at rest and pain on activity," Osteoarthritis and Cartilage, vol. 26, no. 3, pp. 363-369, 2018.

[17] L. Fan, X. Guan, W. Wang et al., "Impaired neuropathic pain and preserved acute pain in rats overexpressing voltage-gated potassium channel subunit Kv1.2 in primary afferent neurons," Molecular Pain, vol. 10, p. 1744, 2014.

[18] J. Y. Zhao, L. Liang, X. Gu et al., "DNA methyltransferase DNMT3a contributes to neuropathic pain by repressing Kcna2 in primary afferent neurons," Nature Communications, vol. 8, no. 1, p. 14712, 2017.

[19] V. Das, R. Kc, X. Li et al., "Pharmacological targeting of the mammalian clock reveals a novel analgesic for osteoarthritisinduced pain," Gene, vol. x655, pp. 1-12, 2018.

[20] R. E. Miller, S. Ishihara, B. Bhattacharyya et al., "Chemogenetic inhibition of pain neurons in a mouse model of osteoarthritis," Arthritis \& Rheumatology, vol. 69, no. 7, pp. 1429-1339, 2017.

[21] N. E. Waly, A. Refaiy, and N. M. Aboreha, "IL-10 and TGF- $\beta$ : roles in chondroprotective effects of Glucosamine in experimental osteoarthritis?" Pathophysiology, vol. 24, no. 1, pp. 45-49, 2017.

[22] C. Otis, J. Gervais, M. Guillot et al., "Concurrent validity of different functional and neuroproteomic pain assessment methods in the rat osteoarthritis monosodium iodoacetate (MIA) model," Arthritis Research \& Therapy, vol. 18, no. 1, p. 150, 2016.

[23] S. Bar-Yehuda, L. Rath-Wolfson, L. Del Valle et al., "Induction of an antiinflammatory effect and prevention of cartilage damage in rat knee osteoarthritis by CF101 treatment," Arthritis Rheum, vol. 60, no. 10, pp. 3061-3071, 2009.

[24] J. Fernihough, C. Gentry, M. Malcangio et al., "Pain related behaviour in two models of osteoarthritis in the rat knee," Pain, vol. 112, no. 1, pp. 83-93, 2004.

[25] L. Meyer, C. Patte-Mensah, O. Taleb, and A. G. MensahNyagan, "Neurosteroid $3 \alpha$-androstanediol efficiently counteracts paclitaxel-induced peripheral neuropathy and painful symptoms," PLoS One, vol. 8, no. 11, Article ID e80915, 2013.
[26] W. Shen, X. M. Hu, Y. N. Liu et al., "CXCL12 in astrocytes contributes to bone cancer pain through CXCR4-mediated neuronal sensitization and glial activation in rat spinal cord," Journal of Neuroinflammation, vol. 11, no. 1, p. 75, 2014.

[27] X. M. Hu, S. B. Cao, H. L. Zhang et al., "Downregulation of miR-219 enhances brain-derived neurotrophic factor production in mouse dorsal root ganglia to mediate morphine analgesic tolerance by upregulating CaMKII $\gamma$," Molecular Pain, vol. 12, no. 47, pp. 14670-14683, 2016.

[28] X. Zhao, Z. Tang, H. Zhang et al., "A long noncoding RNA contributes to neuropathic pain by silencing Kcna2 in primary afferent neurons," Nature Neuroscience, vol. 16, no. 8, pp. 1024-1031, 2013.

[29] Y. Kim, E. H. Kim, K. S. Lee et al., "The effects of intra-articular resiniferatoxin on monosodium iodoacetate-induced osteoarthritic pain in rats," The Korean Journal of Physiology \& Pharmacology, vol. 20, no. 1, pp. 129-136, 2016.

[30] K. Koda, K. Hyakkoku, K. Ogawa et al., "Sensitization of TRPV1 by protein kinase $\mathrm{C}$ in rats with mono-iodoacetateinduced joint pain," Osteoarthritis Cartilage, vol. 24, no. 7, pp. 1254-1262, 2016.

[31] J. Nam, P. Perera, J. Liu et al., "Transcriptome-wide gene regulation by gentle treadmill walking during the progression of monoiodoacetate-induced arthritis," Arthritis \& Rheumatism, vol. 63, no. 6, pp. 1613-1625, 2011.

[32] D. R. Sagar, L. E. Staniaszek, B. N. Okine et al., "Tonic modulation of spinal hyperexcitability by the endocannabinoid receptor system in a rat model of osteoarthritis pain," Arthritis \& Rheumatism, vol. 62, no. 12, pp. 3666-3676, 2010.

[33] P. I. Mapp, D. R. Sagar, S. Ashraf et al., "Differences in structural and pain phenotypes in the sodium monoiodoacetate and meniscal transection models of osteoarthritis," Osteoarthritis Cartilage, vol. 21, no. 9, pp. 1336-1345, 2013.

[34] R. Pelletier, J. Higgins, and D. Bourbonnais, "Addressing neuroplastic changes in distributed areas of the nervous system Associated with chronic musculoskeletal disorders," Physical Therapy, vol. 95, no. 11, pp. 1582-1591, 2015.

[35] I. Albayrak, S. Apiliogullari, C. N. Dal, F. Levendoglu, and O. M. Ozerbil, "Efficacy of pulsed radiofrequency therapy to dorsal root ganglion adding to TENS and exercise for persistent pain after total knee arthroplasty," The Journal of Knee Surgery, vol. 30, no. 2, pp. 134-142, 2017.

[36] H. G. Schaible, F. Richter, A. Ebersberger et al., "Joint pain," Experimental Brain Research, vol. 196, no. 1, pp. 153-162, 2009.

[37] C. J. Woolf and M. Costigan, "Transcriptional and posttranslational plasticity and the generation of inflammatory pain," Proceedings of the National Academy of Sciences, vol. 96, no. 14, pp. 7723-7730, 1999.

[38] D. Lu, J. Chen, and T. Hai, "The regulation of ATF3 gene expression by mitogen-activated protein kinases," Biochemical Journal, vol. 401, no. 2, pp. 559-567, 2007.

[39] T. Hai, C. D. Wolfgang, D. K. Marsee et al., "ATF3 and stress responses,” Gene Expression, vol. 7, no. 4-6, pp. 321-325, 1999.

[40] K. Nobori, H. Ito, M. Tamamori-Adachi et al., “ATF3 inhibits doxorubicin-induced apoptosis in cardiac myocytes: a novel cardioprotective role of ATF3," J Mol Cell Cardiol, vol. 34, no. 10, pp. 1387-1397, 2002.

[41] N. Ohba, M. Maeda, S. Nakagomi et al., "Biphasic expression of activating transcription factor-3 in neurons after cerebral infarction," Molecular Brain Research, vol. 115, no. 2, pp. 147-156, 2003. 
[42] J. S. Francis, M. Dragunow, and M. J. During, "Over expression of ATF-3 protects rat hippocampal neurons from in vivo injection of kainic acid," Molecular Brain Research, vol. 124, no. 2, pp. 199-203, 2004.

[43] H. M. Chen, L. Wang, and S. R. D’Mello, “Inhibition of ATF-3 expression by B-Raf mediates the neuroprotective action of GW5074," Journal of Neurochemistry, vol. 105, no. 4, pp. 1300-1312, 2008.

[44] S. Nakagomi, Y. Suzuki, K. Namikawa, S. Kiryu-Seo, and H. Kiyama, "Expression of the activating transcription factor 3 prevents c-Jun $\mathrm{N}$-terminal kinase-induced neuronal death by promoting heat shock protein 27 expression and Akt activation," The Journal of Neuroscience, vol. 23, no. 12, pp. 5187-5196, 2003.

[45] K. Obata, H. Yamanaka, T. Fukuoka et al., "Contribution of injured and uninjured dorsal root ganglion neurons to pain behavior and the changes in gene expression following chronic constriction injury of the sciatic nerve in rat," Pain, vol. 101, no. 1-2, pp. 65-77, 2003.

[46] C. M. Peters, J. R. Ghilardi, C. P. Keyser et al., "Tumor-induced injury of primary afferent sensory nerve fibers in bone cancer pain," Experimental Neurology, vol. 193, no. 1, pp. 85-100, 2005.

[47] H. Tsujino, E. Kondo, T. Fukuoka et al., "Activating transcription factor 3 (ATF3) induction by axotomy in sensory and motoneurons: a novel neuronal marker of nerve injury," Molecular and Cellular Neuroscience, vol. 15, no. 2, pp. 170$182,2000$.

[48] J. M. Bráz and A. I. Basbaum, "Differential ATF3 expression in dorsal root ganglion neurons reveals the profile of primary afferents engaged by diverse noxious chemical stimuli," Pain, vol. 150, no. 2, pp. 290-301, 2010.

[49] J. J. Inglis, A. Nissim, D. M. Lees, S. P. Hunt, Y. Chernajovsky, and B. L. Kidd, "The differential contribution of tumour necrosis factor to thermal and mechanical hyperalgesia during chronic inflammation," Arthritis Research \& Therapy, vol. 7, no. 4, pp. R807-R816, 2005.

[50] S. Averill, S. B. McMahon, D. O. Clary, L. F. Reichardt, and J. V. Priestley, "Immunocytochemical localization of trkA receptors in chemically identified subgroups of adult rat sensory neurons," European Journal of Neuroscience, vol. 7, no. 7, pp. 1484-1494, 1995.

[51] J. J. Inglis, C. A. Notley, D. Essex et al., "Collagen-induced arthritis as a model of hyperalgesia: functional and cellular analysis of the analgesic actions of tumor necrosis factor blockade," Arthritis \& Rheumatism, vol. 56, no. 12, pp. 4015-4023, 2007.

[52] S. P. Ivanavicius, A. D. Ball, C. G. Heapy, R. F. Westwood, F. Murray, and S. J. Read, "Structural pathology in a rodent model of osteoarthritis is associated with neuropathic pain: increased expression of ATF-3 and pharmacological characterisation," Pain, vol. 128, no. 3, pp. 272-282, 2007.

[53] H. Misonou and J. S. Trimmer, "Determinants of voltagegated potassium channel surface expression and localization in Mammalian neurons," Critical Reviews in Biochemistry and Molecular Biology, vol. 39, no. 3, pp. 125-145, 2004.

[54] E. K. Yang, K. Takimoto, Y. Hayashi, W. C. de Groat, and N. Yoshimura, "Altered expression of potassium channel subunit mRNA and alpha-dendrotoxin sensitivity of potassium currents in rat dorsal root ganglion neurons after axotomy," Neuroscience, vol. 123, no. 4, pp. 867-874, 2004.

[55] Z. Li, Y. Guo, X. Ren et al., "HDAC2, but not HDAC1, regulates Kv1.2 expression to mediate neuropathic pain in CCI rats," Neuroscience, vol. 408, pp. 339-348, 2019.
[56] L. Liang, X. Gu, J. Y. Zhao et al., "G9a participates in nerve injury-induced Kcna2 downregulation in primary sensory neurons," Scientific Reports, vol. 6, no. 1, p. 37704, 2016. 